Notas Clínicas

\title{
Infección VIH y sarcoma de Kaposi: a propósito de un caso
}

\author{
D. Vicente Baz ${ }^{1}$, C. Fernández Delgado²
}

\section{Resumen}

- Propósito: El sarcoma de Kaposi es el cáncer más frecuente entre los pacientes con infección VIH aunque el riesgo ha disminuido con el tratamiento antirretroviral. Realizamos una revisión bibliográfica de esta enfermedad.

- Material y métodos: Se presenta el caso de un varón de 49 años VIH + diagnosticado de sarcoma de Kaposi con afectación visceral y tratado con adriamicina liposomal.

- Conclusiones: Si la enfermedad es sintomática, rápidamente progresiva o existe afectación visceral se debe iniciar quimioterapia.

\section{Palabras clave:}

Sarcoma de Kaposi. Infección VIH. Adriamicina liposomal.

Oncología, 2005; 28 (10):483-486

\footnotetext{
${ }^{1}$ Unidad de Oncología Médica

${ }^{2}$ Unidad de Medicina Interna

Hospital de Palamós-Baix Empordá

Palamós (Girona)
} 


\section{Summary}

- Purpose: Kaposi's sarcoma is the most common type of cancer among the patients with acquired immunodeficiency syndrome. The incidence diminished substantially with the antiretroviral therapy. We study a case and make a bibliographical review of this entity.

- Material and methods: A 49 year old male patient with HIV infection and Kaposi's sarcoma with visceral involvement who was treated with liposomal doxorubicin is presented.

- Conclusion: When the disease is symptomatic, rapidly progressive or shows visceral involvement, a systemic therapy with liposomal anthracycline is advisable.

Key words: Kaposi’s sarcoma. HIV infection. Liposomal doxorubicin.

\section{Introducción}

El Sarcoma de Kaposi es la neoplasia más frecuente en pacientes con infección VIH, sobre todo en homosexuales ${ }^{1}$. Sin embargo, desde principios de los años 90 se ha observado una disminución drástica en la incidencia ${ }^{2}$, coincidente con el desarrollo del tratamiento antirretroviral. También es más frecuente en pacientes trasplantados, apareciendo habitualmente a partir del año del transplante. Muchos casos post-transplante son resultado de una reactivación viral aunque también está descrita la transmisión donante-receptor a través del órgano donado ${ }^{3}$.

Afecta más a varones que a mujeres con ratio de 3,3. La edad media de diagnóstico es a los 43 años, mucho más temprano que en pacientes con Sarcoma de Kaposi clásico.

\section{Caso clínico}

Paciente varón homosexual de 49 años con antecedentes de tuberculosis pulmonar en la infancia tratada con estreptomicina, esfinterotomía lateral interna reciente por fisura anal y pólipos centinela, serología VIH positiva diagnosticada en enero 2003, en estadio C3 por neumonía por Pneumocystis carinii. Último control CD 30 y 2.220 .000 copias virales. Inicia tratamiento con lamivudina-zidovudina-ritonavir-lopinavir. Consulta por dolor epigástrico intenso postpandrial sin pirosis. En la exploración física se aprecian lesiones sobreelevadas rojovioláceas en zona laterocervical izquierda e inguinal derecha, la mayor de $2 \mathrm{~cm}$ de diámetro máximo. Se realiza endoscopia oral, apreciándose lesiones eritematosas en estómago y primeras porciones duodenales, alguna de ellas ulceradas con biopsia positiva para sarcoma de Kaposi. Estudio de extensión negativo, con TAC toracabdominal normal. Con el diagnóstico de sarcoma de kaposi con afectación visceral y cutánea inicia tratamiento con adriamicina liposomal 20 $\mathrm{mg} / \mathrm{m}^{2}$ cada 3 semanas. Desde el primer de ciclo de tratamiento, se produce la desaparición de la afectación cutánea y el paciente queda asintomático, al tercer ciclo se repite endoscopia oral, mostrando una resolución completa de la lesiones.

\section{Discusión}

En 1994 se descubrió el virus Herpes Humano 8 (HHV-8), como agente etiológico del sarcoma de $\mathrm{Kaposi}^{4}$. El HHV-8 es un virus oncogénico que codifica citokinas, celulas involucradas en la proliferación, apoptosis y respuestas inmunes. La forma de transmisión más común es vía sexual, siendo la seroprevalencia de HHV-8 más elevada en varones homosexuales, incrementándose con el número de parejas y en hombres con otras enfermedades de transmisión sexual ${ }^{5}$. Aunque la infección es menos frecuente en mujeres se han detectado niveles más altos en prostitutas que en la población general ${ }^{6}$. Las rutas exactas de transmisión no son bien conocidas, parece que las practicas sexuales con transferencia de fluidos orales, dónde el virus se encuentra más frecuentemente, podrían ser una importante vía de 
transmisión ${ }^{7,8}$. La transferencia por hemoderivados o por contacto con agujas infectadas puede acontecer, pero es menos probable que para otros virus ${ }^{3}$. La transmisión vertical materno-infantil es rara, aunque se han descrito casos ${ }^{9}$.

La preponderancia de casos de sarcoma de Kaposi en ciertos grupos étnicos aparece ligada a la distribución geográfica de la infección por HHV-8 menos del 5\% en USA, norte-Europa y Asia, entre 5-20\% en area del Mediterráneo, medio Este, Caribe y más del $50 \%$ en Africa Central y Sur.

La probabilidad de que un paciente HIV positivo, HHV-8 positivo, desarrolle un sarcoma de Kaposi se sitúa entre el 30\%-50\% ${ }^{10}$, siendo más frecuente en pacientes con recuentos bajos de CD4 o número elevado de copias de $\mathrm{HIV}^{11}$ y en varones homosexuales ${ }^{7}$.

El SK en el $60 \%$ de los casos afecta la piel y la mucosa orofaringea, suele presentarse en forma de lesiones cutáneas sobreelevadas de color rojo-violáceo. El $40 \%$ restante la afectación es visceral, sobretodo del tracto gastrointestinal pulmonar, pudiendo originar hemorragias, perforación u obstrucción. Cuando existe afectación digestiva, las imágenes endoscópicas son bastante características, visulizándose las características lesiones violáceas. Las biopsias mucosas obtenidas por endoscopia no muestran un gran rendimiento diagnostico debido a la fecuente localización submucosa del tumor.

El curso del Sarcoma de Kaposi depende del nivel de inmunosupresión. La supervivencia estimada a los 5 años es del $69 \%$ pero varía dependiendo de la extensión de la enfermedad. La supervivencia en el primer año es del $90 \%$ para la afectación cutánea y del 70\% para la afectación visceral.

La reducción de la inmunosupresión logra una reducción del tumor en el $50 \%$ de los casos, sobre todo en las formas cutáneas. En los casos con afectación visceral o ampliamente diseminados el tratamiento de elección es la quimioterapia. Las respuestas oscilan entre $27-80 \%$ según las series ${ }^{12,13}$. La evaluación de la respuesta en estos pacientes no sigue los criterios oncológicos habituales, pudiendo lograrse la respuesta completa a pesar de la persistencia de las lesiones cutáneas en forma de máculas residuales pigmentadas ${ }^{14}$.

Entre los múltiples agentes utilizados la adriamicina liposomal y el paclitaxe ${ }^{15,16}$ son considerados los agentes más activos. En dos estudios fase III la adriamicina liposomal demostró un mayor índice de respuestas y un mejor perfil de toxicidad que esquemas de poliquimioterapia ${ }^{17,18}$. No se debe olvidar realizar profilaxis frente a Pneumocystis carinii y puede ser necesario instaurar tratamiento con G-CSF.

La utilidad clínica de los fármacos antivirales (foscarnet, ganciclovir, cidafavir y adaafavir) que tienen actividad contra HHV-8 in vitro no está bien establecida.

\author{
Correspondencia: \\ Dr. D. Vicente Baz \\ Unidad de Oncología Médica \\ Hospital Juan Ramón Jiménez \\ Avda. Ronda Norte, s/n \\ E-21006 Huelva \\ dvicentebaz@yahoo.es
}

\section{Bibliografía}

1. Ledergerber B, Telenti A, Egger M. Risk of HIV related Kaposi's sarcoma and non-Hodgkin's lymphoma with potent antiretroviral therapy: Prospective cohort study. BMJ 1999;319:23-24.

2. Jones JL, Hanson DL, Dworkin MS, et al. Incidence and trends in Kaposi's sarcoma in the era of effective antiretroviral therapy. J Acquir Immune defic Syndr 2000;24:270-274.

3. Luppi M, Barozzi P, Santagostino G, et al. Molecular evidence of organ-related transmission of Kaposi sarcoma associated herpesvirus or human herpesvirus- 8 in trasplant patients. Bood 2000;96:3279-81.

4. Chang Y, Cesarman E, Pessin MS, et al. Identification of herpervirus-like DNA sequences in AIDS-associated Kaposi's sarcoma. Science 1994;266:1865-9.

5. Martin JN, Ganem DE, Osmond DH, Page-Shafer KA, Macrae D, Kedes DH. Sexual transimission and the natural history of human herpesvirus 8 infection. N Engl J Med 1998;338:948-54.

6. Cannon MJ, Dollard SC, Smith DK, et al. Bood-borne and sexual transmission of human herpes virus ( in women with or at risk for human immunodeficiency virus infection. $\mathrm{N}$ Engl J Med 2001;344:637-43.

7. Pauk J, Huang ML, Brodie SJ, et al. Mucosal shedding of human herpesvirus 8 in men. N Engl J Med 2000;343: 1369-77.

8. Martin JN, Osmond DH. Invited commentary: determining specific sexual practices associated with human herpesvirus 8 transmission. Am J Epidemiol 2000;15:225-9.

9. Mantina H, Kankasa C, klaskala W, et al. Vertical transmission of kaposi's sarcoma-associated herpesvirus. Int $\mathrm{J}$ Cancer 2001;94:749-52. 
10. Rezza G, Andreoni M, Dorrucci M, et al. Human herpesvirus 8 seropositivity and risk of Kaposi's sarcoma and other acquired immunodeficiency syndrome-related diseases. J Nat Cancer Inst 1999;91:1468-74.

11. Jacobson LP, Jenkins FJ, Springer G, et al. Interaction of human immunodeficiency virus type 1 and human herpesvirus type 8 infections on the incidence of Kaposi's sarcoma. J Infect dis 2000;181:1940-9.

12. Volberding PA, Abrams DI, Conant M, et al. Vinblastine therapy for Kaposi's sarcoma in the acquuired immunodeficiency syndrome. Ann Intern Med 1985;103:335338.

13. Laubenstein LS, Krigel RL, Odajnyk CM, et al. Treatment of epidemic kaposi's sarcoma with etopodide or a combination of doxorubicin, bleomycin and vinblastine. J Clin Oncol 1984;2:1115-1120.

14. Miralles P, Podzamczer D, De la Calle M, et al. Recomendaciones de GESIDA/Plan Nacional sobre el Sida al diagnóstico y tratamiento del sarcoma de Kaposi y el cáncer de cérvix uterino en pacientes infectados por el virus de la inmunodeficiencia humana. Med Clin 2002;118:788-795.

15. Gill PS, Tulpule A, Espina BM, et al. Paclitaxel is safe and effective in the treatment of advanced AIDS-related kapisi's sarcoma. J Clin Oncol 1999;17:1876-1883.

16. Welles L, Saville MW, Lietzau J, et al. Phase II trial with dose titration of paclitaxel for the therapy of human immunodeficiency virus-associated kaposi's sarcoma. J Clin Oncol 1998;16:1112-1121.

17. Northfelt DW, Debuze BJ, Thommes JA, et al. Pegylatedliposomal doxorubicin versus doxorubicin, bleomycin, and vincristine in the treatment of AIDS-related Kaposi's sarcoma: results of a randomized phase III clinical trial. J Clin Oncol 1998;16:2445-51.

18. Stewart S, Jablonowski H, Goebel FD, et al. Randomized comparative trial of pegylated liposomal doxorubicin versus bleomycin and vincristine in the treatment of AIDS-related Kaposi's sarcoma. International Pegylated Liposomal Doxorubicin Study Group. J Clin Oncol 1998;16:638-91. 